\title{
Propiedades físico-mecánicas y simulación por computadora del daño por impacto en guayaba (Psidium guajava L.)
}

\author{
Physical-mechanical properties and impact damage computer simulation in guava (Psidium guajava L.)
}

\author{
Holanda Martínez-Martínez ${ }^{1}$, Artemio Pérez-López ${ }^{1 *}$, Ma. del Rosario Venegas-Ordoñez², \\ Salvador Valle-Guadarrama ${ }^{1}$
}

\begin{abstract}
RESUMEN
Los factores externos a los que están expuestos los frutos después de la cosecha son determinantes en el lapso de tiempo de vida útil como productos en fresco. Se emplearon frutos de guayaba cosechados en dos estados de madurez (50\% amarillo y $100 \%$ amarillo) con la finalidad de estudiar el efecto de los esfuerzos en el tejido cuando son sometidos a daños por impacto, a través de la medición de sus propiedades físicas, químicas y fisiológicas. Además se emplearon herramientas matemáticas como el método numérico del elemento finito (MEF) para conocer el umbral de esfuerzos generados a partir de la altura de caída. Se encontró que los frutos con caída sobre una superficie de madera, a una distancia de 1.0 $\mathrm{m}$, mostraron los cambios físicos, químicos y fisiológicos del tejido, más significativos del daño mecánico en comparación con un piso firme de concreto. Las pruebas de simulación por MEF mostraron que una altura de caída de $0.1 \mathrm{~m}$ ocasiona esfuerzos, en la zona de impacto, mayores a los del límite elástico del material, lo que sugiere un daño irreversible en el tejido a partir de esta altura de caída y que pudiera extenderse hacia una mayor superficie del tejido dependiendo de los factores ambientales e intrínsecos de la especie.
\end{abstract}

PALABRAS CLAVE

propiedades físicas, carga de compresión, módulo de Young, método del elemento finito, daño por impacto

\section{ABSTRACT}

The external factors to which the fruits are exposed after the harvest are decisive in shelf life as fresh products. Guava fruits harvested in two maturity stages (50\% yellow and 100\% yellow) were used in order to study the effect of stress on the tissue when subjected to impact damage, by measuring its physical, chemical and physiological properties. In addition, mathematical tools such as finite element method (MEF) were used to know the stress threshold generated from the fall height. It was found that fruits falling on a wood surface, at a distance of $1.0 \mathrm{~m}$, showed the physical, chemical and physiological changes of the tissue, more significant of mechanical damage compared to a firm concrete floor. MEF simulation tests showed that a drop height of $0.1 \mathrm{~m}$ causes stresses in the impact zone greater than the elastic limit of the material, suggesting irreversible tissue damage from this height and that could extend to a larger tissue surface depending on the environmental and intrinsic factors of the species.

KEYWORDS

physical properties, compression load, Young's module, finite element method, impact damage

\footnotetext{
${ }^{1}$ Departamento de Ingeniería Agroindustrial, Universidad Autónoma Chapingo.

${ }^{2}$ Departamento de Ingeniería Mecánica Agrícola, Universidad Autónoma Chapingo.

* Autor de correspondencia. Carretera México-Texcoco, km. 38.5. 56230 Estado de México, México.

Correo electrónico: aperezl.dia@gmail.com
} 


\section{INTRODUCCIÓN}

La guayaba (Psidium guajava L.) es un fruto con amplia distribución y demanda en el mercado nacional e internacional y una excelente fuente de vitamina $\mathrm{C}$, riboflavina, niacina y minerales (USDA, 2015); sin embargo, también es uno de los frutos más susceptibles a daños. De acuerdo con el SIAP (2016), la producción nacional de guayaba para 2016 fue de alrededor de 309,093.93 t, lo que representó un valor en la producción de 1.3 millones de pesos. La guayaba en fresco cuenta con un periodo de vida de anaquel de 5 a 7 días a temperatura ambiente y de tres semanas a $8{ }^{\circ} \mathrm{C}$ (Mercado-Silva et al., 1998).

Con base en la norma mexicana NMXFF-040-SCFI-2002 de la guayaba en fresco, la buena calidad del fruto para consumo humano implica, en general, buen aspecto con defectos leves, como raspaduras, rozaduras, costras, manchas o quemaduras de sol, que no afecten su conservación y permitan cumplir con los parámetros de selección. Sin embargo, el fruto es sometido a un gran número de eventos que le ocasionan daños mecánicos en la piel, los cuales generan repercusiones económicas importantes y, en ocasiones, son difíciles de detectar a simple vista. Algunos estudios muestran que la pérdida de producto por daño mecánico es de $12 \%$ de la producción total (Kader, 2002; Wills y Golding, 2016).

Con el fin de mantener la calidad de frutos hasta el momento de su consumo, es necesario conocer la naturaleza de la cadena de distribución, desde la producción hasta el consumidor, y definir los elementos que forman parte de esta cadena. Para entender las causas y mecanismos de los daños mecánicos es necesario considerar los siguientes aspectos: a) la fuente, magnitud y naturaleza de la fuerza que lo ocasiona; b) la influencia del material de empaque y el contenedor mismo; c) la influencia del fruto individual o de los frutos vecinos en la modificación de la fuerza, y d) la susceptibilidad del fruto al daño como una función de la madurez, temperatura, tamaño, cultivar, etc. (Van Zeebroeck et al., 2007).

Las situaciones de esfuerzo a las que es sometido un fruto y que generan daño debido a la cantidad de fuerza aplicada, pueden clasificarse, básicamente, en tres tipos: compresión, impacto y vibración (Ragni y Berardinelli, 2001; Berardinelli et al., 2005; Lewis et al., 2008). En la literatura se reportan múltiples formas de estudiar estos tres tipos de daños mecánicos, las cuales van desde lo experimental hasta el uso de he- rramientas matemáticas como el método del elemento finito (MEF), con la finalidad de conocer o predecir los esfuerzos generados y establecer los umbrales de carga que el tejido experimenta ante estos eventos (Sadrnia et al., 2008; Lewis et al., 2007).

Algunas propiedades físicas, como la forma, el tamaño, el volumen, el área superficial, la densidad, la porosidad, la velocidad terminal, el color y la apariencia, son empleadas como parámetros ingenieriles en el diseño de equipo de manejo poscosecha. Por su parte, las propiedades mecánicas, como la deformación, el esfuerzo, el esfuerzo de corte, la fuerza de compresión, el limite elástico, el módulo de elasticidad, la razón de poisson, el punto de cedencia, el límite proporcional, el punto de biocedencia, la rigidez, el esfuerzo de relajación, etc., se han empleado para definir la respuesta de un fruto ante la acción de una fuerza compresiva o tensional (ASAE, 2005; Szczesniak, 1983).

Este trabajo de investigación propone estudiar el efecto de los esfuerzos en el tejido de frutos de guayaba, sometidos a daños por impacto, a través de la medición de sus propiedades físico-químicas y fisiológicas. Además de emplear herramientas computacionales acopladas a la herramienta matemática del método numérico del elemento finito (MEF), para entender el comportamiento de los esfuerzos del tejido interno del fruto en un escenario de simulación virtual para establecer el umbral de esfuerzos generados a partir de la altura caída del fruto.

\section{Materiales Y MÉTODOS}

Material vegetal. Se emplearon frutos de guayaba cosechados en dos estados de madurez, con base en el color de la superficie externa: 50\% amarillo y $100 \%$ amarillo. Los frutos fueron trasladados a la Universidad Autónoma Chapingo, Texcoco, Estado de México. La caracterización física y fisiológica se realizó en el laboratorio de Fisicoquímica del Departamento de Ingeniería Agroindustrial, mientras que las propiedades mecánicas se determinaron en el Laboratorio de Materiales del Departamento de Ingeniería Mecánica Agrícola de la Universidad Autónoma Chapingo.

Diseño experimental. Las pruebas de compresión se realizaron conforme a un diseño completamente al azar, formando lotes de 10 frutos, cada lote representó un estado de madurez ( $50 \%$ amarillo y $100 \%$ amarillo) de cosecha. La carga de compresión fue aplicada en 
fruto entero y en una porción de pulpa del fruto, en la forma de un prisma rectangular, obtenida con una navaja de corte. En las pruebas de impacto, el diseño se realizó completamente al azar en arreglo factorial $2 \times 2 \times 3 \times 6$ (cuatro factores: estado de madurez, altura de caída, superficie de impacto y tiempo de observación). Se consideraron las alturas de caída más frecuentes en las etapas de manejo poscosecha, antes de llegar al mercado de venta final. Los tratamientos fueron: 1) estado de madurez: $50 \%$ amarilla y $100 \%$ amarilla; 2 ) altura de caída: $0,0.5$ y $1 \mathrm{~m}$; 3) superficie de impacto: concreto y madera, y 4) tiempo de evaluación: 0, 12, $24,36,48$ y $60 \mathrm{~h}$. Las variables consideradas en las pruebas de impacto y las variables fisiológicas se midieron con tres repeticiones a la temperatura del ambiente durante el periodo de almacenamiento.

Propiedades físicas. Se midieron longitud $(L)$, diámetro mayor $(m)$ y diámetro menor $(n)$ (figura 1 ) con un calibrador digital marca Trupper ${ }^{\circledR}$ CALDI-6MP. Con estas variables se calcularon el diámetro medio geométrico (ecuación 1), la esfericidad (ecuación 2) (Mohsenin, 1986) y el área superficial (ecuación 3) de la fruta (McCabe et al., 2001).

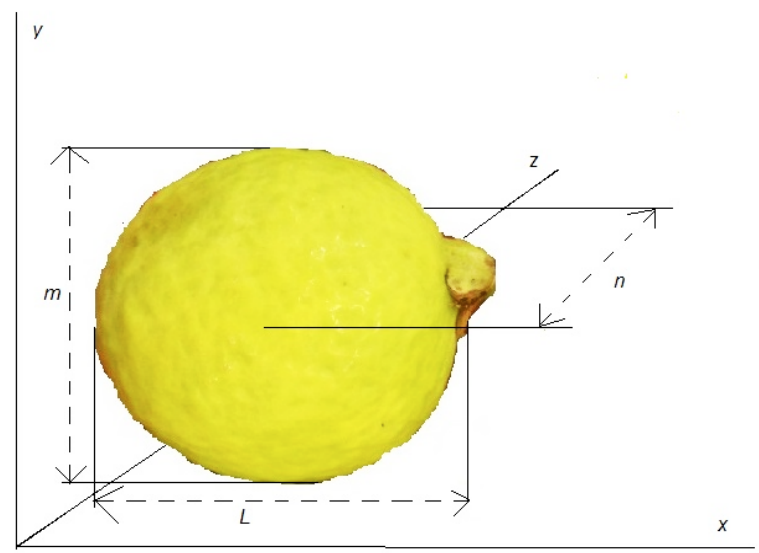

Figura 1. Representación esquemática de la longitud ( $L$ ), diámetro mayor $(m)$ y diámetro menor $(n)$ del fruto de guayaba.

$$
\begin{aligned}
& D_{g}=(L \cdot m \cdot n)^{\frac{1}{3}} \\
& \varphi=\frac{D_{g}}{L}
\end{aligned}
$$

$$
A_{s}=\pi D_{g}^{2}
$$

La densidad del fruto se calculó con la ecuación 4 . Para ello, se medió el peso de cada fruto con una balanza electrónica digital $\left(\mathrm{OHAUS}^{\circledR}, \mathrm{EUA}\right)$ y se calculó el volumen por el método de desplazamiento de agua, utilizando un recipiente de volumen y peso conocidos. Se empleó como referencia la densidad del agua a la temperatura de $20{ }^{\circ} \mathrm{C}\left(0.998 \mathrm{~g} \cdot \mathrm{cm}^{-3}\right.$ (Rafiee et al., 2007). Para la determinación de solidos solubles totales, se usó un refractómetro portátil $\left(\mathrm{ATAGO}^{\circledR}\right.$, Japón).

$$
\rho_{f}=\frac{W_{f}}{V_{f}}
$$

Propiedades mecánicas. Se aplicaron pruebas de compresión uniaxial para obtener las curvas de fuerza-deformación (ASAE, 2005). A partir de estas gráficas, se analizó la región lineal (región elástica) y se calcularon las variables carga de compresión en kilonewton $(\mathrm{kN})$, porcentaje de deformación (\%) y el módulo de Young en Megapascales (MPa). El equipo utilizado fue una maquina universal de ensayos mecánicos INSTRON (modelo 3382, Instron, Norwood, MA, EUA) enlazada a una computadora personal por medio del software Bluehill ${ }^{\circledR} 2$ de INSTRON. La velocidad de desplazamiento de la cruceta del equipo fue de $50 \mathrm{~mm} \cdot \mathrm{min}^{-1}$ y se empleó una celda de carga de $100 \mathrm{kN}$ (Pérez-López et al., 2014). La carga de compresión fue aplicada en el fruto entero y en una porción del tejido en forma de prisma rectangular (figura 2). El fruto entero se colocó

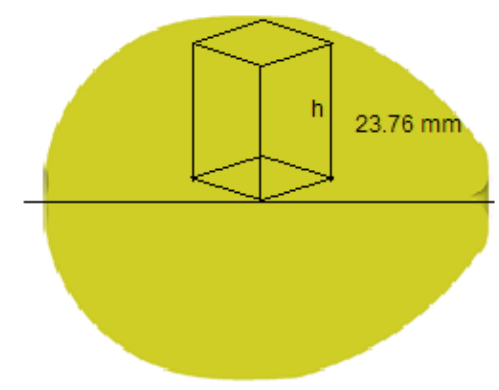

Figura 2. Representación esquemática de la sección donde se obtuvo el prisma rectangular de tejido del fruto de guayaba. 
en posición horizontal con el pedúnculo-cáliz de forma paralela al plato de compresión y, en el prisma rectangular, la carga de compresión $(\mathrm{kN})$ se aplicó en la dirección longitudinal de la muestra.

Pruebas de impacto. Se determinó el cambio de color del fruto sometido a impacto a través de un espectrofotómetro de esfera (X-rite ${ }^{\circledR}$, Mod. SP64, EUA), usando la escala CIEL ${ }^{*} a^{*} b^{*}$. Se midieron los valores de $L^{*}, a^{*} y b^{*}$. Con estos valores se calcularon la luminosidad $\left(\mathrm{L}^{*}\right)$, el ángulo de tono (hue), la pureza de color (Chroma), además del cambio total de color $(\Delta \mathrm{E})$ de acuerdo con la ecuación 5.

$$
\Delta E=\sqrt{\left(\Delta L^{*}\right)^{2}+\left(\Delta a^{*}\right)^{2}+\left(\Delta b^{*}\right)^{2}}
$$

El peso individual de los frutos se midió con una balanza digital (OHAUS $\left.{ }^{\circledR}, \mathrm{EUA}\right)$ y con ello se calculó la pérdida de peso, expresada como porcentaje de pérdida diaria con respecto al día inicial.
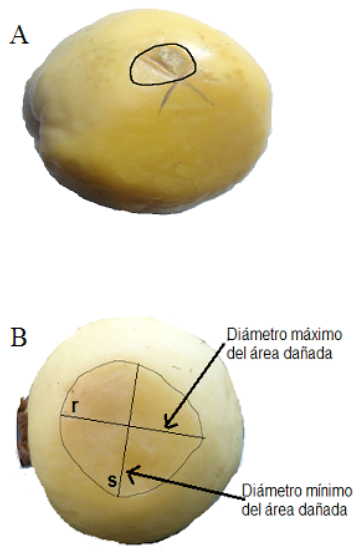

C

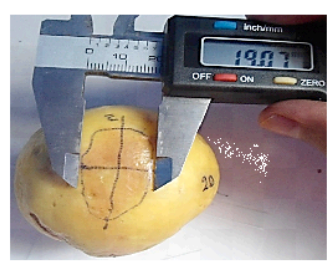

$\mathrm{D}$

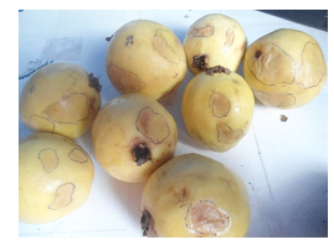

Figura 3. Medición de las áreas dañadas del fruto de guayaba. Identificación del punto de impacto $(A)$, reconocimiento de la superficie dañada (B), medición de la superficie dañada (C) y evaluación del porcentaje de daño (D).

El daño visual se midió empleando la metodología propuesta por Menesatti y Paglia (2001). Con ayuda del calibrador digital (Trupper ${ }^{\circledR}$ ) CALDI-6MP, se tomaron medidas en dos ejes perpendiculares (diámetro menor y diámetro mayor) de la zona dañada (por lo general de forma elíptica) (figura 3) y con ello se calculó el área dañada $A=r * s * \pi$. Con ayuda de una cámara SONY (modelo Cyber-Shot W610, Sony Co., Japón), se tomaron fotografías a las 0, 36 y 60 horas para estimar la evolución del daño.

Pruebas fisiológicas. La velocidad de respiración del fruto $\left(R_{f}\right)$ se evaluó mediante el método estático empleando un sensor de $\mathrm{CO}_{2}$ Vernier LABQUEST (Vernier Software \& Technology, Beaverton, OR, USA). Se tomaron las lecturas de concentración de $\mathrm{CO}_{2}$ en los tiempos $t_{1}$ y $t_{2}$, se midió el volumen libre $(\mathrm{mL})$ del recipiente y la masa del fruto $\left(m_{f}\right)$ en kilogramos. La velocidad de respiración se cuantificó como $\mathrm{mL} \cdot \mathrm{CO}_{2} \cdot \mathrm{kg}^{-1} \cdot \mathrm{h}^{-1}$ de acuerdo con la ecuación 6 .

$$
R_{f}=\frac{\left(\left[\mathrm{CO}_{2}\right]_{t_{2}}-\left[\mathrm{CO}_{2}\right]_{t_{1}}\right)}{m_{f} \cdot(\Delta t)}
$$

Modelado sólido tridimensional de la guayaba en computadora. El modelo sólido 3D del fruto fue desarrollado en el software comercial de modelado de sólidos paramétrico 3D Solidworks Simulation (SolidWorks v.2013, Waltham, Mass, EUA). Un fruto de guayaba fue cortado exactamente a la mitad de su geometría en la orientación perpendicular al eje cáliz-pedúnculo y se dibujó sobre papel el contorno de la superficie externa de una mitad de la pieza cortada, con una secuencia muy fina de puntos sobre

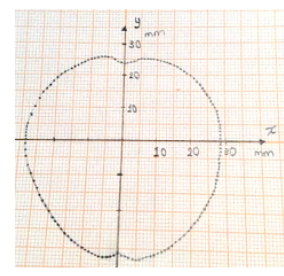

A)

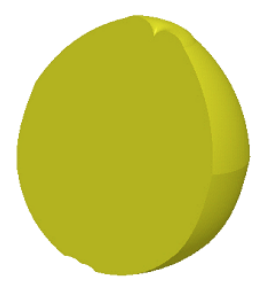

B)

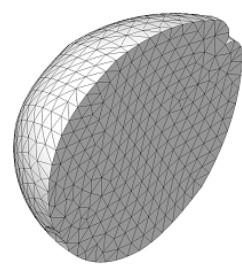

C)
Figura 4. A) Contorno del fruto en un sistema de coordenadas bidimensional, B) Modelo 3D de la geometría de la mitad del fruto de guayaba y C) Mallado con elementos sólidos tetrahédricos de segundo orden en el modelo 3D del fruto.

la línea que describe el contorno; sus coordenadas se midieron en el plano bidimensional $x$-y (figura 4A). Mediante este sistema de coordenadas de los puntos y con el empleo del software de matemáticas MatLab R2013a (Mathworks, Inc., Massachusetts, 
EUA), se obtuvo la ecuación polinomial que describe la geometría de la superficie. Se creó un modelo 3D de la mitad de la geometría del fruto (figura 4B) y el mallado se construyó en el mallador automático de Solidworks Simulation con el cual se generaron elementos sólidos tetrahédricos de segundo orden. Se obtuvo un total de 10,360 elementos con un total de 11,554 nodos (figura 4C).

Simulación del impacto. Antes de la simulación, se introdujeron las propiedades del material en el módulo de simulación del software. Se eligió como zona de impacto la región ecuatorial del fruto y se probaron distancias de caída de 100, 50, 30, 10 y $5 \mathrm{~cm}$. El tiempo de solución después del impacto se calculó de forma automática con el software y se estableció en 17.22 microsegundos. Se definieron 25 intervalos de tiempo (número de trazados) después del primer momento de impacto durante la simulación.

Análisis estadístico. En las variables físicas y fisiológicas, se calculó el promedio de tres repeticiones con su respectiva desviación estándar. En la evaluación visual se reportó el porcentaje de área dañada con su respectiva barra de error estándar. Las variables de propiedades mecánicas, color, pérdida de peso y daño visual se valoraron mediante análisis de varianza multifactorial con la prueba Tukey $(\alpha \leq 0.05)$. Los análisis estadísticos se realizaron con el software estadístico Statistical Analysis System (SAS Institute Inc., Cary, EUA).

\section{RESULTADOS Y DiscuSIóN}

Propiedades físicas. Los valores del diámetro medio geométrico $\left(\mathrm{D}_{\mathrm{g}}\right)$, esfericidad $(\phi)$ y el área superficial $\left(\mathrm{A}_{\mathrm{s}}\right)$ de los frutos verdes fueron 57.8, 0.91 y 105.5; para los frutos amarillos fueron 55.9, 0.92, 98, respectivamente (cuadro 1). No se observó diferencia estadística significativa entre ambos estados de madurez. La variabilidad en las propiedades físicas depende de varios factores; entre ellos, la variedad, las condiciones del ambiente de producción, el manejo agronómico y nutricional del cultivo, etc. Un estudio realizado con guayaba de la variedad híbrido 'Enana roja' EEA-1-23 reportó diámetro medio ecuatorial de 60.0 a $70.23 \mathrm{~mm}$ en cuatro estados de madurez (Rodríguez et al., 2012).

Propiedades mecánicas. La carga de compresión para alcanzar el limite elástico fue significativa $(p \leq 0.05)$, en el factor estado de madurez, donde tuvo valores de
Cuadro 1. Propiedades físicas del fruto de la guayaba en dos estados de madurez (media \pm desviación estándar, $n=50$ ).

\begin{tabular}{|c|c|c|}
\hline Propiedad & $\begin{array}{c}50 \% \\
\text { AMARILLA }\end{array}$ & $\begin{array}{c}100 \% \\
\text { AMARILLA }\end{array}$ \\
\hline Longitud $(L), \mathrm{mm}$ & $63.49 \pm 3.1$ & $60.56 \pm 3.1$ \\
\hline $\begin{array}{l}\text { Diámetro mayor }(m), \\
m m\end{array}$ & $55.44 \pm 2.6$ & $53.79 \pm 2.9$ \\
\hline $\begin{array}{l}\text { Diámetro menor }(n) \text {, } \\
\text { mm }\end{array}$ & $55.11 \pm 2.4$ & $53.71 \pm 3.2$ \\
\hline $\begin{array}{l}\text { Diámetro medio } \\
\text { geométrico }(\mathrm{D} g), \mathrm{mm}\end{array}$ & $57.89 \pm 2.2$ & $55.91 \pm 2.6$ \\
\hline Esfericidad, \% & $0.91 \pm 0.0$ & $0.92 \pm 0.0$ \\
\hline $\begin{array}{l}\text { Área de la superficie, } \\
\mathrm{cm}^{2}\end{array}$ & $105.57 \pm 8.1$ & $98.4 \pm 95.4$ \\
\hline Peso, g & $98.64 \pm 13.8$ & $93.17 \pm 14.3$ \\
\hline Volumen, $\mathrm{cm}^{3}$ & $95.44 \pm 10.4$ & $90.76 \pm 12.3$ \\
\hline Densidad, $\mathrm{g} \cdot \mathrm{cm}^{-3}$ & $1.04 \pm 0.0$ & $1.03 \pm 0.0$ \\
\hline${ }^{\circ}$ Brix, \% & $10.8 \pm 0.45$ & $11.45 \pm 0.33$ \\
\hline
\end{tabular}

Cuadro 2. Análisis factorial para carga de compresión, esfuerzo, deformación y módulo de Young del tejido de frutos de guayaba.

\begin{tabular}{lcccc}
\hline FACTOR & CARGA & & Defor- & Módulo \\
NIVEL & DE & ESFUERzo & MACIÓN & DE \\
& COMPRE- & & $\begin{array}{c}\text { Young } \\
\text { (MPA) }\end{array}$ \\
& SIÓN $(K N)$ & & & \\
\hline
\end{tabular}

Estado de

madurez

(A)

\begin{tabular}{|c|c|c|c|c|}
\hline $\begin{array}{l}50 \% \\
\text { amarilla }\end{array}$ & $124.44^{\mathrm{a}}$ & $65.36^{\mathrm{a}}$ & $37.41^{\mathrm{a}}$ & $0.19^{a}$ \\
\hline $\begin{array}{l}100 \% \\
\text { amarilla }\end{array}$ & $75.32^{\mathrm{b}}$ & $65.35^{\mathrm{a}}$ & $38.78^{a}$ & $0.17^{a}$ \\
\hline HSD & 42.26 & 28.63 & 7.24 & 0.08 \\
\hline \multicolumn{5}{|c|}{$\begin{array}{l}\text { Geometría } \\
\text { de fruto } \\
\text { (B) }\end{array}$} \\
\hline $\begin{array}{l}\text { Fruto } \\
\text { entero }\end{array}$ & $186.93^{a}$ & $58.73^{\mathrm{a}}$ & $37.34^{\mathrm{a}}$ & $0.16^{\mathrm{a}}$ \\
\hline $\begin{array}{l}\text { Prisma } \\
\text { rectan- } \\
\text { gular }\end{array}$ & $12.83^{\mathrm{b}}$ & $71.98^{\mathrm{a}}$ & $38.85^{\mathrm{a}}$ & $0.21^{\mathrm{a}}$ \\
\hline HSD & 42.26 & 28.63 & 7.24 & 0.08 \\
\hline $\mathrm{CV}$ & 65.94 & 68.31 & 29.66 & 67.67 \\
\hline$A^{*} B$ & $7.14^{*}$ & $6.79 *$ & $0.50^{\text {ns }}$ & $3.31^{\mathrm{ns}}$ \\
\hline
\end{tabular}

Diferentes superíndices dentro de la misma columna que corresponden a un factor indican que las medias difieren significativamente (Tukey, $\alpha=0.05$ ); (ns) estadísticamente no significativo; ( ) estadísticamente significativo al $P \leq 0.05$. 
Cuadro 3. Análisis multifactorial para porcentaje de pérdida de peso (\% PP), luminosidad (L), ángulo de tono (hue), pureza de color (croma) y cambio de color $(\Delta \mathrm{E})$ del fruto de guayaba.

\begin{tabular}{|c|c|c|c|c|c|}
\hline FACTOR NIVEL & $\%$ PP & $\mathbf{L}$ & hue $\left(^{\circ}\right)$ & Снroma & $\Delta \mathbf{E}$ \\
\hline \multicolumn{6}{|l|}{ Estado de madurez (A) } \\
\hline $50 \%$ amarilla & $2.69^{a}$ & $72.77^{\mathrm{b}}$ & $89.19^{a}$ & $52.73^{\mathrm{a}}$ & $9.73^{a}$ \\
\hline $100 \%$ amarilla & $2.66^{\mathrm{a}}$ & $77.92^{\mathrm{a}}$ & $86.27^{\mathrm{b}}$ & $53.30^{\mathrm{a}}$ & $5.40^{\mathrm{b}}$ \\
\hline HSD & 0.19 & 1.66 & 0.53 & 0.83 & 0.67 \\
\hline \multicolumn{6}{|l|}{ Superficie de impacto (B) } \\
\hline Concreto & $2.64^{\mathrm{a}}$ & $74.93^{\mathrm{a}}$ & $87.74^{\mathrm{a}}$ & $53.23^{\mathrm{a}}$ & $7.48^{\mathrm{a}}$ \\
\hline Madera & $2.71^{\mathrm{a}}$ & $75.96^{\mathrm{a}}$ & $87.72^{\mathrm{a}}$ & $52.69^{a}$ & $7.68^{\mathrm{a}}$ \\
\hline HSD & 0.19 & 1.69 & 0.54 & 0.85 & 0.68 \\
\hline \multicolumn{6}{|l|}{ Altura de impacto (C) } \\
\hline $0 \mathrm{~m}$ & $2.40^{\mathrm{b}}$ & $76.00^{\mathrm{a}}$ & $86.99^{b}$ & $53.05 \mathrm{ab}$ & $7.50^{\mathrm{b}}$ \\
\hline $0.5 \mathrm{~m}$ & $2.73^{\mathrm{a}}$ & $74.73^{\mathrm{a}}$ & $88.62^{\mathrm{a}}$ & $54.08 \mathrm{a}$ & $5.90^{c}$ \\
\hline $1.0 \mathrm{~m}$ & $2.62^{\mathrm{a}}$ & $75.63^{\mathrm{a}}$ & $87.22^{\mathrm{b}}$ & $51.93 b$ & $9.25^{\mathrm{a}}$ \\
\hline HSD & 0.19 & 2.57 & 0.82 & 1.29 & 1.0 \\
\hline \multicolumn{6}{|l|}{ Tiempo de observación (D) } \\
\hline $0 \mathrm{~h}$ & $0.00^{\mathrm{f}}$ & $71.95^{\mathrm{c}}$ & $93.06^{\mathrm{a}}$ & $50.86^{\mathrm{d}}$ & $0.00^{\mathrm{f}}$ \\
\hline $12 \mathrm{~h}$ & $1.38^{\mathrm{e}}$ & $73.33^{\mathrm{bc}}$ & $90.88^{\mathrm{b}}$ & $51.59^{\mathrm{cd}}$ & $4.11^{\mathrm{e}}$ \\
\hline $24 \mathrm{~h}$ & $2.03^{\mathrm{d}}$ & $75.56^{\mathrm{abc}}$ & $87.63^{c}$ & $52.24^{\mathrm{cd}}$ & $7.25^{\mathrm{d}}$ \\
\hline $36 \mathrm{~h}$ & $3.11^{\mathrm{c}}$ & $75.96^{\mathrm{abc}}$ & $85.76^{\mathrm{d}}$ & $53.17^{\mathrm{bc}}$ & $9.61^{\mathrm{c}}$ \\
\hline $48 \mathrm{~h}$ & $4.22^{\mathrm{b}}$ & $77.17^{\mathrm{ab}}$ & $85.09^{\text {de }}$ & $54.52^{\mathrm{ab}}$ & $11.33^{\mathrm{b}}$ \\
\hline $60 \mathrm{~h}$ & $5.32^{\mathrm{a}}$ & $78.09^{a}$ & $83.96^{\mathrm{e}}$ & $55.70^{\mathrm{a}}$ & $13.06^{\mathrm{a}}$ \\
\hline HSD & 0.5 & 4.21 & 1.34 & 2.12 & 1.70 \\
\hline $\mathrm{CV}$ & 22.42 & 7.47 & 2.05 & 5.35 & 30.05 \\
\hline$A^{*} B$ & $3.42^{\text {ns }}$ & $6.58^{*}$ & $5.23^{*}$ & $15.71^{* * *}$ & $1.43^{\text {ns }}$ \\
\hline$A^{*} \mathrm{C}$ & $14.77^{* * *}$ & $0.39^{\mathrm{ns}}$ & $14.52^{* * *}$ & $4.16^{*}$ & $17.62^{* * *}$ \\
\hline$A^{*} D$ & $0.94^{\mathrm{ns}}$ & $2.93^{*}$ & $17.15^{* * *}$ & $1.95^{\mathrm{ns}}$ & $13.48^{* * *}$ \\
\hline $\mathrm{B}^{*} \mathrm{C}$ & $3.10^{\text {ns }}$ & $4.33^{*}$ & $4.37^{*}$ & $0.88^{\text {ns }}$ & $2.22^{\text {ns }}$ \\
\hline$B^{*} \mathrm{D}$ & $0.08^{\mathrm{ns}}$ & $1.07^{\mathrm{ns}}$ & $0.55^{\mathrm{ns}}$ & $0.08^{\mathrm{ns}}$ & $0.13^{\text {ns }}$ \\
\hline$C^{*} \mathrm{D}$ & $1.88^{\mathrm{ns}}$ & $1.29^{\mathrm{ns}}$ & $6.32^{* * *}$ & $0.11^{\mathrm{ns}}$ & $4.23^{* * *}$ \\
\hline$A^{*} B^{*} C^{*} D$ & $1.63^{\text {ns }}$ & $1.03^{\text {ns }}$ & $1.06^{\mathrm{ns}}$ & $0.89^{\text {ns }}$ & $1.16^{\mathrm{ns}}$ \\
\hline
\end{tabular}

Diferentes superíndices dentro de la misma columna que corresponden a un factor indican que las medias difieren significativamente (Tukey, $\alpha=0.05)$; (ns) estadísticamente no significativo; $\left({ }^{*},{ }^{* * *}\right)$ estadísticamente significativo al $P \leq 0.05$ y 0.001 , respectivamente.

$124.4 \mathrm{kN}$ y $75.3 \mathrm{kN}$ para los frutos verde y amarillo, respectivamente. Lo mismo sucedió para el factor geometría del fruto, donde el fruto entero alcanzó carga máxima de $186.9 \mathrm{kN}$ hasta el límite elástico. Las propiedades de esfuerzo, deformación y módulo de Young no fueron significativas estadísticamente; sin embargo, la rigidez del tejido fue mayor en los frutos $50 \%$ amarillos. El efecto de interacciones de los factores estado de madurez y geometría de fruto en la compresión mostró diferencia significativa en las variables carga de compresión y esfuerzo (cuadro 2 ). 
Pruebas de impacto. El cambio de color, durante el almacenamiento, en la mayoría de todos los tratamientos mostró diferencias significativas $(P \leq 0.05)$. El aumento en el valor de $L^{*}$ para la fruta amarilla indica un mayor brillo en su superficie, y cambio de amarillo claro a amarillo rojizo por la disminución de hue y croma (cuadro 3). Los cambios de color se presentaron progresivamente durante los días de almacenamiento y se hicieron notables a las 60 h después del impacto, con un factor de cambio en el color de 13.06. Según Wang y Mellenthin (1973), el cambio en la coloración se debe a una combinación de estrés físico y reacciones bioquímicas causadas por la oxidación de los compuestos fenólicos. En cuanto al porcentaje de peso perdido se encontraron diferencias significativas $(P \leq 0.05)$ en el factor altura de impacto, entre el testigo y los frutos con impacto (cuadro 3 ).

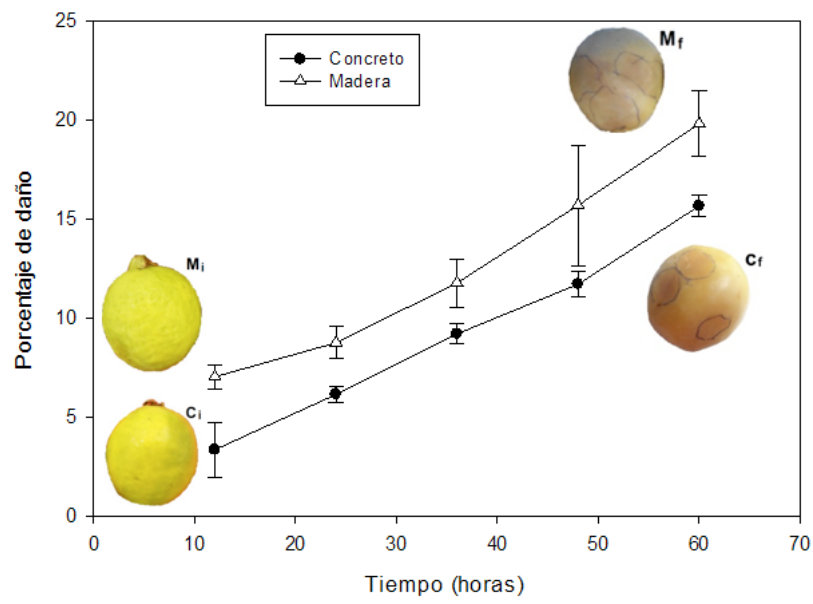

Figura 5. Porcentaje de daño en la guayaba sometida a impacto a una altura de $1 \mathrm{~m}$ sobre dos superficies (concreto y madera), después de $60 \mathrm{~h}$ de almacenamiento. Cada punto representa la media de tres repeticiones \pm error estándar.

Evolución del daño visual en la prueba de impacto. Se encontraron diferencias entre las dos superficies de impacto: las guayabas que se dejaron caer sobre una superficie de madera mostraron más porcentaje de daño que las que cayeron sobre superficie de concreto. El daño visual se caracterizó por coloración oscura (figura 5); es decir, la caída sobre las dos superficies (concreto y madera) provocó oscurecimiento de la epidermis del fruto, el cual se extendió a las células del mesocarpio. Se observó oscurecimiento de mayor intensidad en impacto sobre superficie de madera, con $19.77 \%$ de daño después de $60 \mathrm{~h}$ de almacenamiento. Lo anterior puede atribuirse a la influencia de la rugosidad del material de impacto.

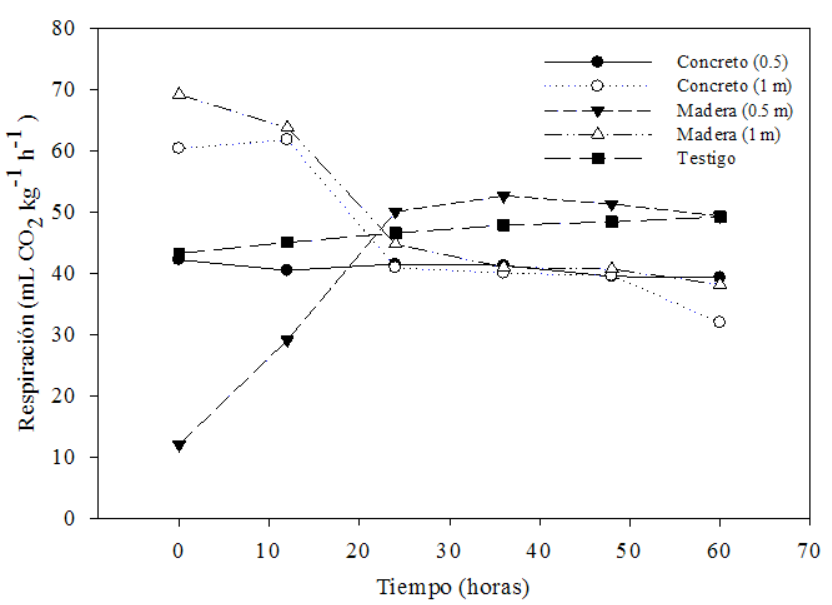

(A)

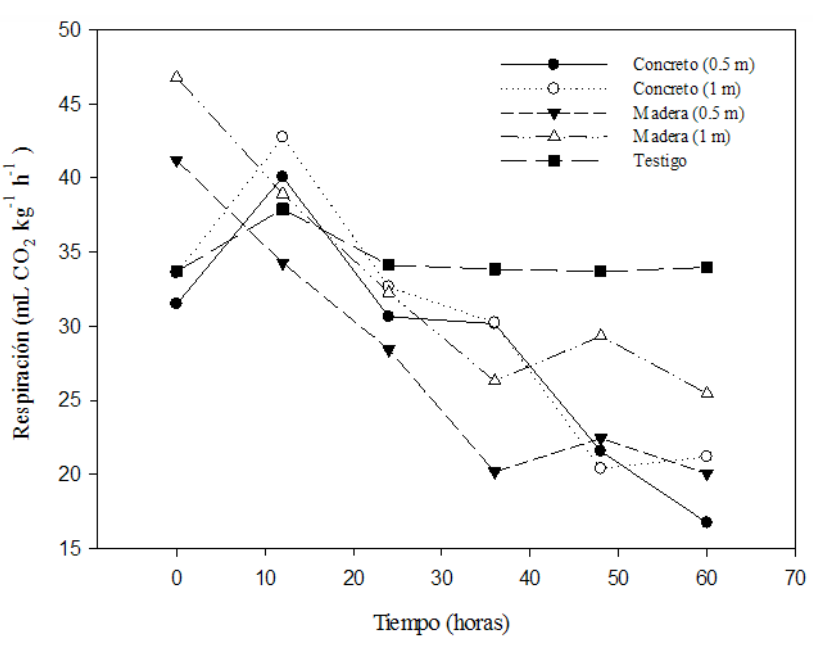

(B)

Figura 6. Variación en la tasa de respiración de la guayaba almacenada $60 \mathrm{~h}$ en estado de madurez fisiológica $50 \%$ amarilla (A) y $100 \%$ amarilla (B) sometida a impacto a una altura de $0.5 \mathrm{~m}$ y $1 \mathrm{~m}$ sobre dos superficies (concreto y madera) y comparada contra un testigo. Cada punto representa la media de tres repeticiones \pm error estándar.

La magnitud del daño por impacto en frutos depende de la velocidad de impacto o altura de caída -además de las características físicas de la superficie de caída- y disminuye como resultado del impacto contra la superficie de un material blando (O'Brien y Gaffney, 1983).

Caracterización fisiológica. Los frutos con 50\% amarillo que no fueron sometidos a daño por impacto no mostraron cambios significativos (44-50 $\left.\mathrm{mL} \cdot \mathrm{CO}_{2} \cdot \mathrm{kg}^{-1} \cdot \mathrm{h}^{-1}\right)$ en la velocidad de respiración durante $60 \mathrm{~h}$ de almacenamiento (figura 6A). La velocidad de respiración fue mayor al incrementar la altura de caída: los frutos que cayeron de $1.0 \mathrm{~m}$ sobre 


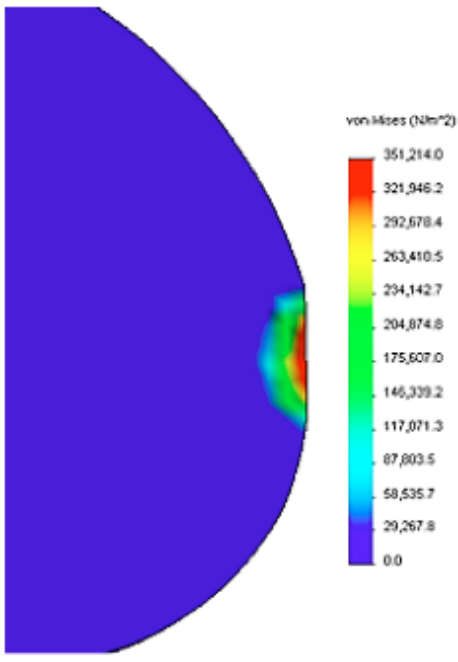

$100 \mathrm{~cm}$

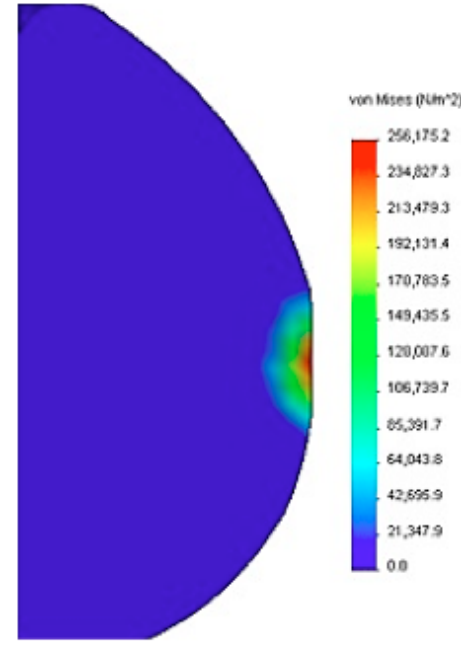

$50 \mathrm{~cm}$

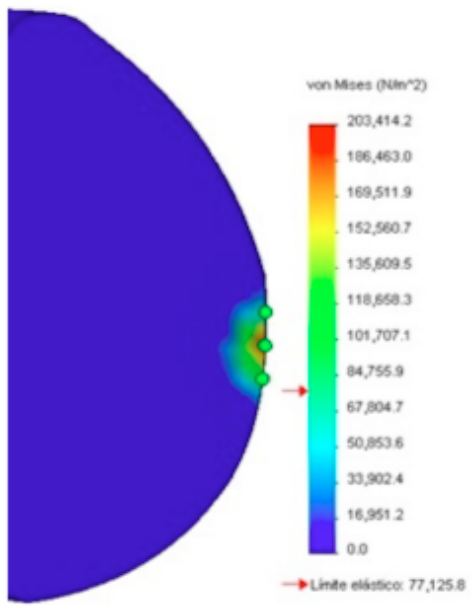

$30 \mathrm{~cm}$

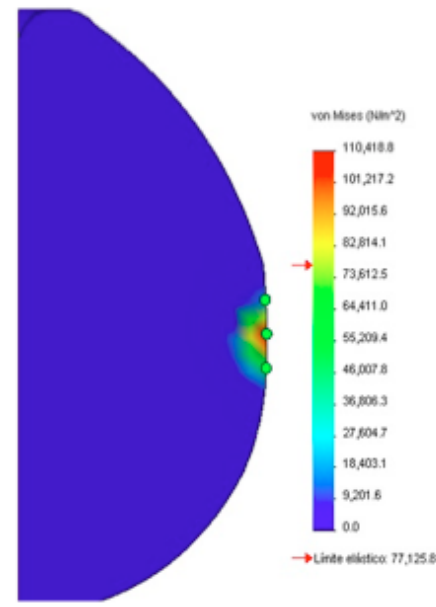

$10 \mathrm{~cm}$

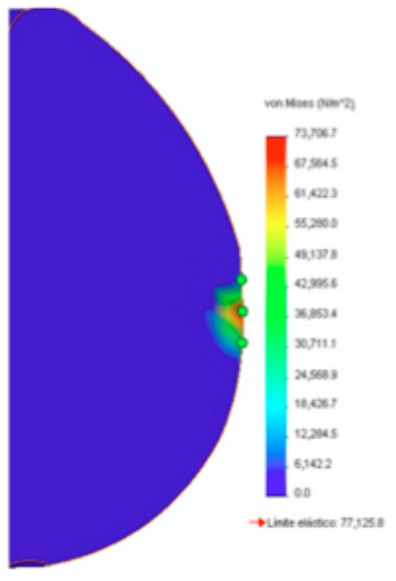

$5 \mathrm{~cm}$

Figura 7. Esfuerzo de impacto en los nodos correspondientes a la zona de impacto en las diferentes alturas de caída del fruto durante el tiempo de solución de la simulación.

superficie de concreto y madera mostraron 17 y $25 \%$ más respiración que los frutos testigo. Los frutos 100\% amarillo mostraron un metabolismo con tendencia a la senescencia durante el periodo de almacenamiento (figura 6B).

Sin embargo, aunque no se detectó gran diferencia en los frutos que cayeron a $0.5 \mathrm{~m}$ sobre superficie de madera, éstos respiraron $7 \%$ más que los frutos testigo. Estudios realizados en zapote mamey (Pouteria sapota), cosechados en diferentes meses (mayo y junio) y sometidos a impacto a diferentes alturas $(0.0,0.6,1.2$ y $1.8 \mathrm{~m}$ ) mostraron que la velocidad de respiración fue mayor al incrementar la altura. Los frutos que cayeron a 1.20 y $1.80 \mathrm{~m}$ mostraron 27 y 15\% más respiración que el testigo (Hernández et al., 2008). El incremento en respiración se atribuye al hecho de que, en tejidos dañados, el oxígeno se difunde más rápidamente en el interior de las células y su actividad metabólica se incrementa (Watada et al., 1996).

Simulación del impacto por el método del elemento finito. El área de mayor daño, señalada de color rojo en la simulación (figura 7), con una altura de caída de $100 \mathrm{~cm}$, recibe un esfuerzo de $351.2 \mathrm{kPa}$ y 
Cuadro 4. Análisis factorial para carga de compresión, esfuerzo, deformación y módulo de Young del tejido de frutos de guayaba.

\begin{tabular}{|c|c|c|c|}
\hline \multirow{2}{*}{$\begin{array}{l}\text { Alturu de caída } \\
(\mathrm{cm})\end{array}$} & \multicolumn{2}{|c|}{$\begin{array}{c}\text { Propiedades mecánicas de la } \\
\text { SIMULACIÓN MEF }\end{array}$} & \multirow{2}{*}{$\begin{array}{c}\begin{array}{c}\text { PROPIEDADES MECÁNICAS } \\
\text { POR COMPRESIÓN AXIAL* }\end{array} \\
\begin{array}{c}\text { ESFUERZO A LIMITE ELÁsTICO } \\
\left(\mathrm{N} \cdot \mathrm{m}^{-2} \mathbf{x} 10^{3}\right)\end{array}\end{array}$} \\
\hline & $\begin{array}{c}\text { ESFUERZO EQUIVALENTE } \\
\text { MÁx. } \\
\left(\mathbf{N} \cdot \mathbf{m}^{-2} \mathbf{x} 10^{3}\right)\end{array}$ & $\begin{array}{c}\text { DEFORMACIÓN MÁx. } \\
(\mathrm{mm})\end{array}$ & \\
\hline 100 & 351.2 & 0.025 & 77.1 \\
\hline 50 & 256.1 & 0.017 & 77.1 \\
\hline 30 & 203.4 & 0.012 & 77.1 \\
\hline 10 & 110.4 & 0.006 & 77.1 \\
\hline 5 & 73.7 & 0.004 & 77.1 \\
\hline
\end{tabular}

* Estos valores son de referencia y fueron obtenidos mediante pruebas de compresión axial de fruto entero en una máquina INSTRON.

a $50 \mathrm{~cm}$ de altura de caída este esfuerzo es de 256.1 $\mathrm{kPa}$. A medida que la altura de caída disminuye, los esfuerzos en los nodos de la zona de impacto también disminuyen, de tal manera que, cuando la altura fue de $5 \mathrm{~cm}$, el esfuerzo máximo en la zona de impacto fue de $73.7 \mathrm{kPa}$ (cuadro 4). En frutos de manzana Celik et al. (2011) reportaron valores de esfuerzo máximo de $416 \mathrm{kPa}$ en pruebas de simulación con altura de caída de $0.5 \mathrm{~m}$. Mediante las pruebas de compresión axial se encontró que el tejido de frutos de guayaba alcanzó un esfuerzo máximo en la región lineal o elástica de 77. $1 \mathrm{kPa}$ (cuadro 4).

Desde el punto de vista mecánico, esto significa que un esfuerzo, producto de una carga compresiva o de impacto que rebasa este límite permisible, ocasiona daño irreversible en el tejido del fruto. En el cuadro 4 se observa un decremento en los valores de esfuerzo máximo conforme disminuye la altura de caída del fruto. Una altura de caída de 0.1-1.0 m, respecto del piso de impacto, provoca esfuerzos que están por encima del valor de esfuerzo correspondiente límite elástico del tejido de los frutos. Esto significa que existe una probabilidad muy alta de que las células del tejido del fruto de guayaba hayan experimentado daño estructural irreversible $\mathrm{y}$, consecuentemente, cambios en su metabolismo global (Moshenin, 1986; Knee and Miller, 2002).

El tejido de los frutos que recibe un esfuerzo mecánico, ya sea por carga estática o dinámica, puede tener daño estructural permanente dependiendo de la intensidad del esfuerzo. El efecto inmediato es la oxidación de los compuestos fenólicos por la enzima catecol oxidasa, que demanda un incremento en el consumo de oxígeno (Knee and Miller, 2002; Kader, 2002; Berardinelli et al., 2005).

\section{Conclusiones}

Los frutos de guayaba con caída sobre madera a una distancia de $1.0 \mathrm{~m}$ reflejaron valores altos en las variables color, pérdida de peso y porcentaje de daño. Consecuentemente, este impacto se reflejó en un incremento de hasta $25 \%$ más en la velocidad de respiración en relación con los frutos que no fueron sometidos a daño. La evolución del área dañada alcanzó 19.7\%. Las pruebas de simulación, con herramientas de elemento finito mostraron que, a una altura de caída de $0.1 \mathrm{~m}$, el esfuerzo que alcanza el tejido en el área de impacto está por encima del valor del límite elástico del tejido del fruto $(77.1 \mathrm{kPa})$. Esto sugiere que en las operaciones de manejo poscosecha el tejido del área de impacto comienza a experimentar daño irreversible a partir de estas alturas de impacto. Conviene resaltar que también influyen otros factores como geometría del fruto, estado de madurez, superficie de impacto y variedad. 


\section{LiTERATURA CITADA}

American Society for Agricultural Engineering (ASAE). 2005. Standard S368.4 DEC00: Compression Test of Food Materials of Convex Shape. pp. 601-608. In: ASAE. Standards 2005. 52 ${ }^{\text {nd }}$ ed. Standards Engineering Practives Data. St. Joseph, USA.

Berardinelli, A., V. Donati, A. Giunchi, A. Guarnieri, L. Ragni. 2005. Damage to pears caused by simulated transport. Journal of Food Engineering 66: 219-226.

Celik, H. K., A. E. W. Rennie, I. Akinci. 2011. Deformation behavior simulation of an apple under drop case by finite element method. Journal of Food Engineering 104: 293-298.

Hernández M., M., A. Martínez M., I. Alia T., L. U. Hernández H., R. Osorio O., M. T. Colinas L., V. López M., S. Bautista B., S. Valle G. 2008. Estrés por impacto en frutos de zapote mamey (Pouteria sapota) durante el manejo postcosecha. Revista Fitotecnia Mexicana 31 (3): 61-66.

Kader, A. A. 2002. Postharvest Technology of Horticultural Crops. University of California. Oakland, USA. 535 pp.

Knee, M., R. Miller. 2002. Mechanical Injury. pp. 157-179. In: M. Knee (Ed.). Fruit Quality and its Biological Basis. Sheffield Academic Press Ltd. Sheffield, UK.

Lewis, R., A. Yoxall, L. A. Canty, E. R. Romo. 2007. Development of engineering design tools to help reduce apple bruising. Journal of Food Engineering 83: 356-365.

Lewis, R., A. Yoxall, M. B. Marshall, L. A. Canty. 2008. Characterizing pressure and bruise in apple fruit. Wear 264: 37-46.

McCabe, W., J. Smith, P. Harriott. 2001. Unit Operations of Chemical Engineering. The McGraw-Hill Companies Inc. New York, USA. 1168 pp.

Mercado-Silva, E., P. Benito-Bautista, Ma. de los A. García-Velasco. 1998. Fruits development harvest index and ripening changes of guavas produced in central Mexico. Postharvest Biology and Technology 13: 143150.

Menesatti, P., G. Paglia. 2001. Development of a drop damage index of fruit resistance to damage. Journal of Agricultural Engineering Research 80 (1): 53d64.

Mohsenin, N., 1970. Physical Properties of Plant and Animal Materials. Gordon and Breach Science Publisher. New York, USA. 742 pp.

Mohsenin, N. N. 1986. Physical Properties of Plant and Animal Materials; Structure, Physical Characteristics and Mechanical Properties. Gordon and Breach Science Publishers, New York, USA. 891 pp.

NMX-FF-040-SCFI-2002. Norma para productos alimenticios no industrializados para consumo humano - fruta fresca - guayaba (Psidium guajava L.) - especificaciones. Diario Oficial de la Federación, 10 de junio de 1982. 14 pp.

O'Brien, M., J. J. Gaffney. 1983. Postharvest Handling and Transport Operations. pp. 413-470. In: O’Brien, M., B. F. Cargill, R. B. Fridley (Ed.s). Principles and Practices for Harvesting and Handling Fruits and Nuts. AVI/Van Nostrand. New York, USA.
Pérez-López, A., S. H. Chávez-Franco, C. A. Villaseñor-Perea, T. Espinosa-Solares, L. H. Hernández-Gómez, C. Lobato-Calleros. 2014. Respiration rate and mechanical properties of peach fruit during storage at three maturity stages. Journal of Food Engineering 142:111-117.

Rafiee, S., J. M. Keramat, A. Jafari, M. Sharifi, R. Mirasheh, H. Mobli. 2007. Determining some physical properties of bergamot (Citrus medica). International Agrophysics 21: 293-297.

Ragni, L., A. Berardinelli. 2001. Mechanical behavior of apples and damage during sorting and packaging. Journal of Agricultural Engineering Research 78 (3): 273-279.

Rodríguez G., A., L. Mounson L., A. Hernández G. 2012. Determinación de la firmeza de la guayaba (Psidium guajava L.) en diferentes estados de maduración. Revista Ingeniería Agrícola 2 (1): 42-46.

Sadrnia, H., A. Rajabipour, A. Jafari, A. Javadi, Y. Mostofi, J. Kafashan, E. Dintwa, J. De Baerdemaeker. 2008. Internal bruising prediction in watermelon compression using nonlinear models. Journal of Food Engineering 86: 272-280.

SAS Institute Inc. 1996. SAS/STAT Software: Changes and Enhancements through Release 6.12. SAS Institute Inc. Cary, USA. 1162 pp.

Servicio de Información Agroalimentaria y Pesquera (SIAP). 2016. Anuario Estadístico de la Producción Agrícola. http://infosiap.siap.gob.mx/aagricola_siap_ gb/icultivo/index.jsp

Solidworks 1995-2016. Solidworks on-line help. Dassault Systèmes SolidWorks Corporation. Mass., USA.

Szczesniak A. S. 1983. Physical Properties of Foods: what they are and their Relation to other Food Properties. pp 1-41. In: M. Peleg, E. A. Bagley (Eds.) Physical Properties of Food. Westport, CT: AVI Publishing Co.

United States Department of Agriculture (USDA). 2015. Servicios de investigación agrícola: Consulta de información nutrimental y manejo de frutas y verduras. Disponible en:

www.abcdefrutasyverduras.com/curso/consulta.php?sltFrutas $=86 \&$ tipoCons $=$ in

Wang, C. Y., W. M. Mellenthin. 1973. Relationship of friction discoloration to phenolic compounds in " $\mathrm{d}$ 'Anjou" pears. HortScience 8 (4): 321-323.

Watada, A. E., N. P. Ko, D. A. Minott.1996. Factors affecting quality of fresh-cut horticultural products. Postharvest Biology and Technology 9: 115-125.

Wills, R., J. Golding. 2016. An Introduction to the Physiology and Handling of Fruit and Vegetables. CABI. Sydney, Australia. 304 pp.

Van Zeebroeck, M., V. Van linden, H. Ramon, J. De Baerdemaeker, B. M. Nicola1, E. Tijskens. 2007. Impact damage of apples during transport and handling. Postharvest Biology and Technology 45: 157-167. 